\title{
The Cosmic Ray Air Fluorescence Fresnel lens Telescope (CRAFFT) for the next generation UHECR observatory
}

\section{Mashu YAMAMOTO*}

Shinshu University, Nagano, JAPAN

E-mail: 17w2101kdshinshu-u.ac.70

Yuichiro TAMEDA ${ }^{1}$, Takayuki TOMIDA ${ }^{2}$, Motoki HAYASHI ${ }^{2}$, Daisuke IKEDA ${ }^{3}$, Toshihiro FU $\mathrm{JII}^{3}$, Katsuya YAMAZAKI ${ }^{4}$, Hirokazu IWAKURA ${ }^{2}$

${ }^{1}$ Osaka Electro-Communication University, Osaka, JAPAN

${ }^{2}$ Shinshu University, Nagano, JAPAN

${ }^{3}$ Institute for Cosmic Ray Research, The University of Tokyo, Chiba, JAPAN

${ }^{4}$ Earthquake Research Institute, The University of Tokyo, Tokyo, JAPAN

The flux of the ultra high energy cosmic rays (UHECRs) is extremely low, hence we need a huge observatory for UHECR research. On the other hand, the larger the scale of the observatory, the higher the construction cost. In order to launch the next generation UHECR observatory, we should reduce their construction cost, dramatically. Therefore, we are developing a simple structure fluorescence telescope. The telescope consists of a $1 \mathrm{~m}^{2}$ Fresnel lens, an 8 inch photomultiplier tube, a UV transmitting filter, and an FADC board. We will report the status and the result of the test observation at the Telescope Array site.

35th International Cosmic Ray Conference - ICRC2017

10-20 July, 2017

Bexco, Busan, Korea

\footnotetext{
* Speaker.
} 


\section{Introduction}

The origin of ultra high energy cosmic rays (UHECRs) is one of the mysteries of astroparticle physics. Currently, the Telescope Array Experiment (TA)[W],[]] and the Pierre Auger Observatory (Auger) [B] have observed UHECRs. To explore their sources, it is necessary to measure the energy spectrum, the mass composition, and arrival directions of UHECRs.

The flux of cosmic rays decreases as cosmic ray's energy increases, hence it is not realistic to observe UHECR directly. Therefore, TA and Auger observe UHECRs indirectly using the extensive air shower generated when the cosmic ray interacts with the Earth's atmosphere. In order to observe air showers, they use two types of detectors. One is a surface detector (SD) that measure the number of particles at the ground. Another is a fluorescence detector (FD) that can measure the energy calorimetrically, and the longitudinal development of air showers.

The flux of UHECRs is extremely low, hence we need a huge observatory to increase statistics. FD is a mass composition sensitive detector, but construction cost of FD is higher than that of SD. If the cost of FD decreases, it is useful to construct a huge array of FDs.

Currently, Cosmic Ray Air Fluorescence Fresnel lens Telescope (CRAFFT)[团] and the Fluorescence detector Array of Single-pixel Telescopes (FAST)[B] are under development to reduce the cost. We report the current states and the result of test observation of CRAFFT.

\section{Detector}

CRAFFT is an FD of which structure is very simple. The assembled CRAFFT telescope is shown in Fig.W. The components of CRAFFT telescope are a $1 \mathrm{~m}^{2}$ Fresnel lens, a UV transmitting filter, an 8 inch photomultiplier tube (PMT), an FADC board, and an aluminum frame. The specifications of the components are listed in Table.W. When we operate CRAFFT, the frame of the PMT mount and the Fresnel lens mount are covered to prevent incident light. The field of view (F.O.V.) of CRAFFT is configured to 8 degrees using the spatial filter, which is equivalent to $1 / 4$ of the F.O.V. of a TA telescope. A spot of the moon focused on the focal plane is shown in Fig.D. The diameter of the spot of the moon is about $1 \mathrm{~cm}$ at the focal plane. The transparencies of the Fresnel lens and the UV transmitting filter are measured and almost $90 \%$ between 300 and $400 \mathrm{~nm}$ of wavelength. The total cost is $1 / 10$ or less than current typical FDs.

In addition, the telescope will be operated remotely and automatically. When we construct a huge detector array using FD, it is necessary to observe automatically. We are developing a power supply system and protective lens cover mechanism for the telescope.

\section{Detection Efficiency}

Detection efficiency is estimated by detector simulation of CRAFFT. This simulation includes the detector configuration of CRAFFT. Vertical air showers are generated by Gaisser-Hillas function, and the distance between an air shower core and the detector is from 1 to $40 \mathrm{~km}$. We consider fluorescence and Cherenkov light as emitted light from air showers, and Rayleigh-scattering and 


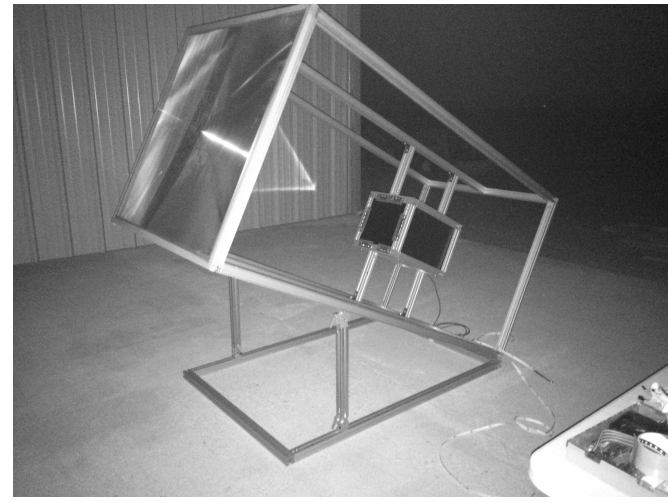

Figure 1: Assembled CRAFFT.

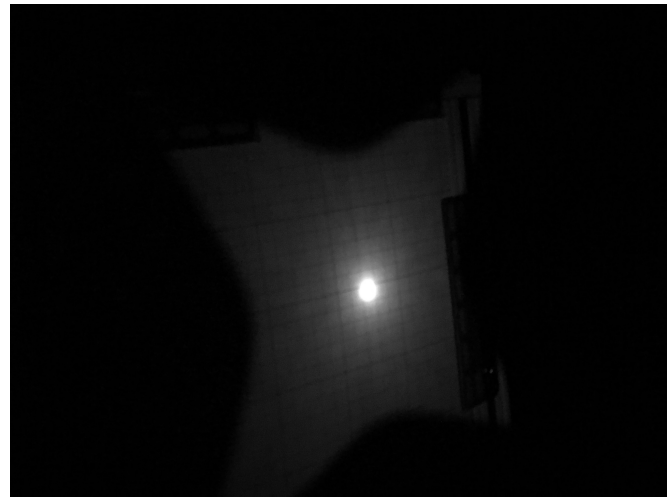

Figure 2: The spot of the moon.

\begin{tabular}{|l|l|l|r|}
\hline Component & Product & Specification & Cost [\$] \\
\hline \hline Fresnel lens & NTKJ, F1200-B & $1 \mathrm{~m}^{2}, \mathrm{f}=1.2 \mathrm{~m}$ & 208 \\
\hline UV transmitting filter & O.M.G., UL-330 & $90 \%, 300-360 \mathrm{~nm}$ & 2667 \\
\hline Photomultiplier tube & hamamatsu, R5912 & 8 inch & 1708 \\
\hline HV power supply & CAEN, N1470AR & $8 \mathrm{kV}, 3 \mathrm{~mA}$ & 1375 \\
\hline FADC & TokushuDenshiKairo, Cosmo-Z & $80 \mathrm{MHz}, 12$ bit & 258 \\
\hline Structure & MIWA & Aluminum frame & 833 \\
\hline \hline Total & & & 7049 \\
\hline
\end{tabular}

Table 1: Specification of the components of CRAFFT.

Mie-scattering for both attenuation and scattering. We assumed the US standard atmosphere model, and the night sky background referred to TA-FD observation. Simulated waveforms are shown in Fig. [3, and simulated $\mathrm{S} / \mathrm{N}$ is in Fig.⿴囗. When $\mathrm{S} / \mathrm{N}$ is greater than $4 \sigma$, CRAFFT telescope can observe the air shower, e.g. CRAFFT can observe $10^{20} \mathrm{eV}$ air shower within $25 \mathrm{~km}$ distance from the telescope.
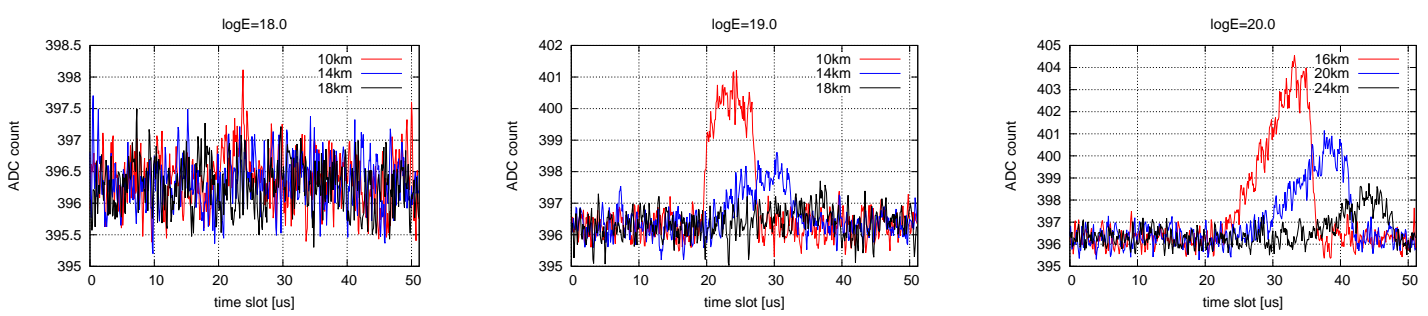

Figure 3: Simulated waveform of CRAFFT for three energies of $10^{19}, 10^{20}, 10^{21} \mathrm{eV}$.

\section{Detector performance test}

We confirmed the performance of the CRAFFT detector by observing the laser shots by the Central Laser Facility (CLF)[6], at TA-FD site in November, 2016. The laser is used for TA-FD calibration as pseudo air shower events with energy of $10^{20} \mathrm{eV}$. 


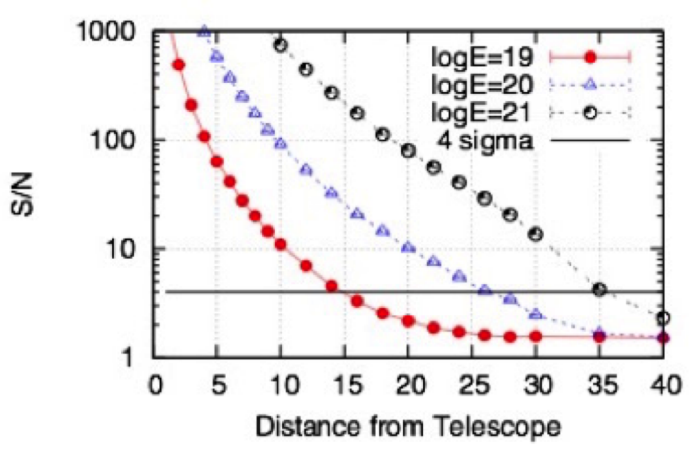

Figure 4: Estimated $\mathrm{S} / \mathrm{N}$ ratio by simulation as a function of distance $[\mathrm{km}]$.

In the early tests, we operated the telescope from the positions 2, 5, and $10 \mathrm{~km}$ from CLF. A map of these locations are shown in Fig.[1. When we transfer the detector to the other point, we put the telescope in the cargo bed of the truck and we operate the telescope as shown in Fig.6. We adjusted azimuth angle of the telescope by a compass and the elevation angle was set to 25 degrees. The power was supplied by a portable generator (YAMAHA, EF900iS). In this observation, we used an oscilloscope instead of the FADC board. The Gain of the amplifier was 17.2. The voltage applied to the PMT was $700 \mathrm{~V}$. We used 1 PPS signal from GPS module as trigger timing, because the laser shooting is synchronized with the GPS. Waveforms of laser shots observed by CRAFFT at 2 and $5 \mathrm{~km}$ points are shown in Fig.Z. Those waveforms are averaged. At $10 \mathrm{~km}$, the laser events couldn't be observed due to night sky background noise by a supermoon.

To evaluate the performance of the detector, we should observe CLF laser from the TA-FD site. We estimated the suitable configuration to observe CLF at the TA-FD site by using simulation because the distance between CLF and the TA-FD site is $20 \mathrm{~km}$ which is longer than the longest distance of the previous test. In the simulation, we estimate the number of photons from the CLF laser energy. Then, we consider attenuation and scattering by Rayleigh and Mie scattering, and the US standard atmosphere for the atmospheric model. Next, scattered photons in the F.O.V. of the telescope are incident on the focal plane through the Fresnel lens and the UV transmitting filter, and transparencies of those are taken into account. Finally, arriving photons are converted to photoelectrons at the photocathode of the PMT. The result of the simulation is shown in Fig.రl. The vertical axis of the graph is the number of photoelectrons that were generated on the photocathode, and the horizontal axis is the elevation angle of the telescope. The assumed distances are 2, 5, 10 , and $20 \mathrm{~km}$ as the TA-FD site. From the simulation, the expected number of photoelectrons observed by the CRAFFT telescope at the TA-FD site is about 70 times less than the number of photoelectrons observed at $2 \mathrm{~km}$. As a result of these calculations, we set the PMT gain to $2 \times 10^{6}$ that is 100 times higher than the previous configuration of $2 \mathrm{~km}$ observation. We succeeded in observing the CLF laser at the TA-FD site. Fig.9, and Fig. 10 are observed waveforms and averaged waveform. There are the spike signals caused by scattering by a cloud in both of waveforms, and the signal before the spike can be identified as normal CLF laser event. 


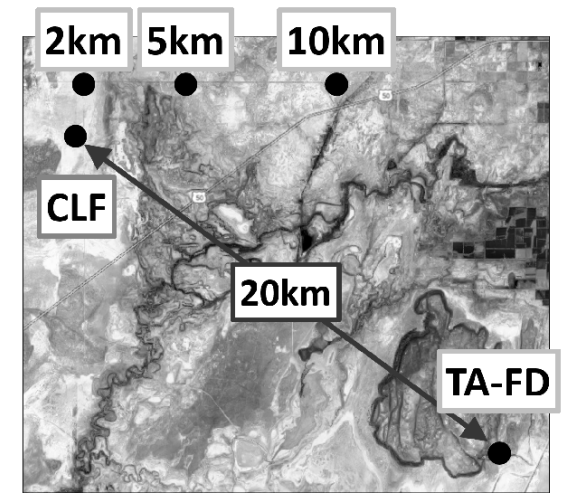

Figure 5: Map of observation positions.

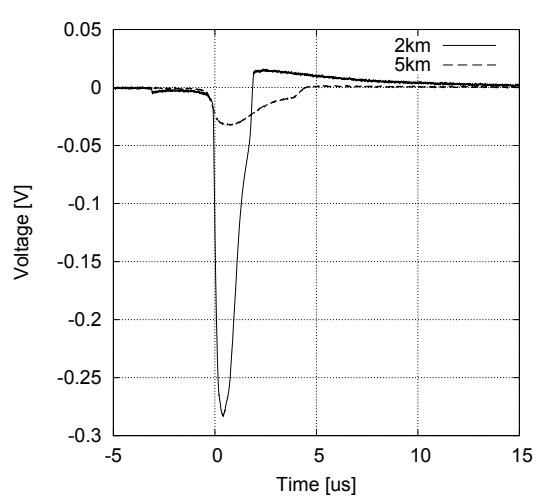

Figure 7: Averaged waveforms of 2, $5 \mathrm{~km}$ (2 $\mathrm{km}: 464$ shots, $5 \mathrm{~km}: 426$ shots).

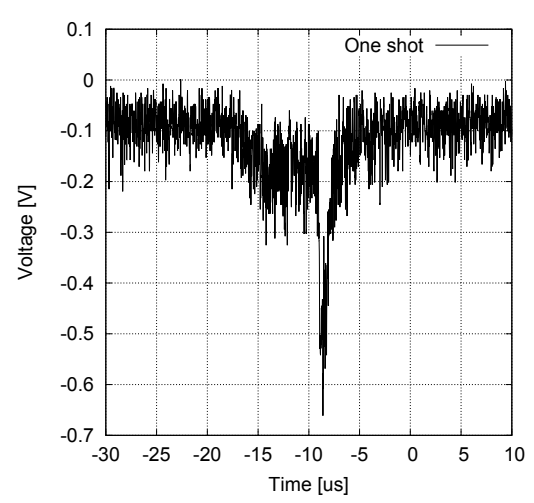

Figure 9: Example of 1 shot waveform.

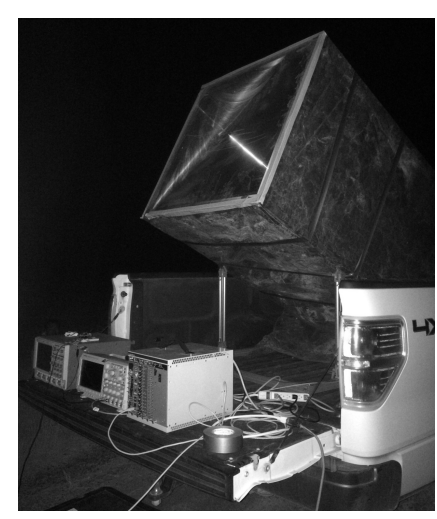

Figure 6: Photo of CRAFFT telescope operating from the bed of a pickup truck.

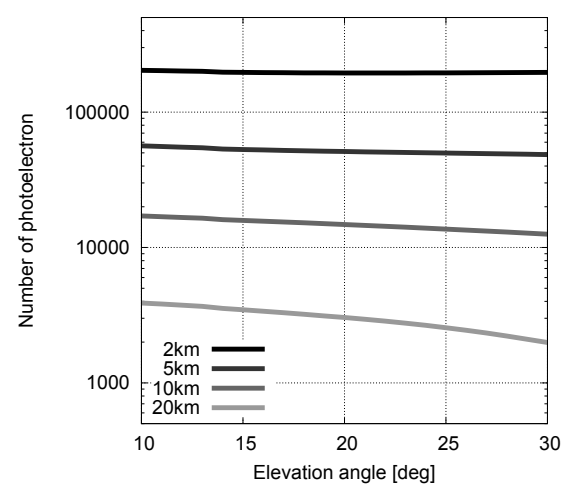

Figure 8: Expected number of photoelectrons.

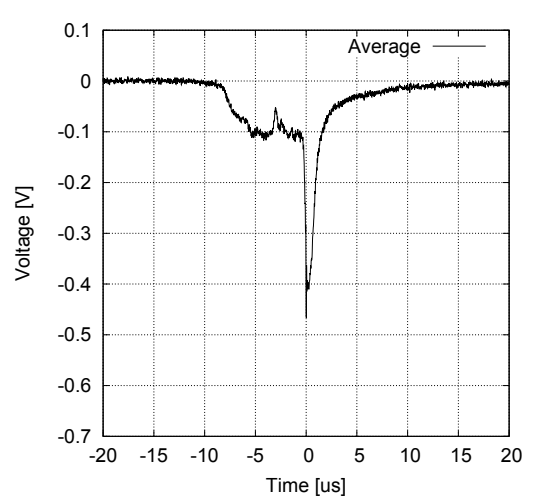

Figure 10: Averaged waveforms(136 shots). 


\section{Trial observation for cosmic ray detection}

We tried to observe cosmic rays at TA-FD site in March, 2017. The elevation angle is 25 degrees. The F.O.V. of CRAFFT was set to the upper view of the TA-FDs. The gain of the amplifier was 17.2. We used the low pass filter of $15 \mathrm{MHz}$ cutoff to reduce the night sky back ground noise. The voltage applied to the PMT was $1250 \mathrm{~V}$. The CRAFFT telescope was triggered by the TA-FD triggering timing from the electronics of TA-FD. We acquired 65,520 waveforms during two nights. The total time of observation was 13 hours. It was clear night to partly cloudy. We calculated the $\mathrm{S} / \mathrm{N}$ of various time windows to search for air shower events. The time windows are 1.6, 3.2, 6.4 and $12.8 \mu \mathrm{s}$. The typical pulse width is assumed to be about $7 \mu$ s considering cosmic rays with energy of $10^{19} \mathrm{eV}$ at $10 \mathrm{~km}$ apart from detector. We extracted waveforms of which the S/N of 6.4 $\mu \mathrm{s}$ is higher than $7 \sigma$. We extracted 1756 waveforms among all of the observed waveforms. The expected number of triggered events is 1365 by considering the ratio of the F.O.V. of CRAFFT and TA-FD, and they are consistent. We found three candidates (Fig.W) of which waveforms are similar to a shape of air shower event. These signals could be air shower events that occurred close to our telescope because the pulse width is narrow.
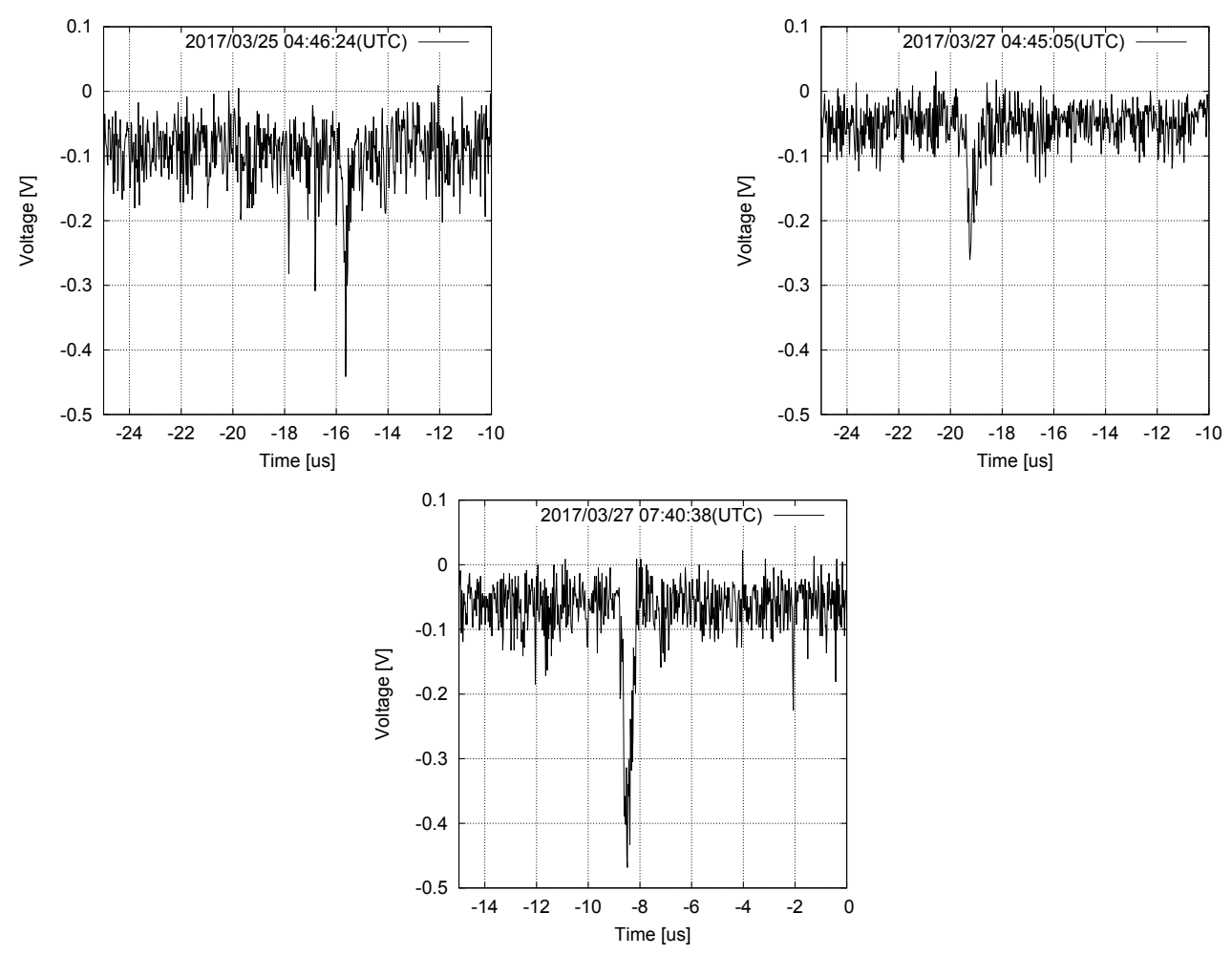

Figure 11: Observed event-like signals. 


\section{Summary}

We are developing a low cost fluorescence detector using Fresnel lens named as Cosmic Ray Air Fluorescence Fresnel lens Telescope (CRAFFT). We aim cost reduction and automatic observation system in order to construct a huge observatory for UHECR research. The cost of prototype telescope is 1/10 of typical FDs. We succeeded in observing the laser events as pseudo air shower events corresponding to the energy of $10^{20} \mathrm{eV}$ by using CLF. Furthermore, we tried to observe cosmic rays in March, 2017 at TA-FD site. There are 3 event candidates.

We are planning three months observation in this summer. We will install 4 CRAFFT telescopes at the TA site.

\section{Acknowledgement}

This work was supported by JSPS KAKENHI Grant Number 25610051 and JP16K17710. This work was partially carried out by the joint research program of the Institute for Cosmic Ray Research (ICRR), the University of Tokyo. the Telescope Array experiment and the members provided us the observation site, trigger, power, network and so on. I am deeply grateful to the TA Collaborators.

\section{References}

[1] H. Tokuno, Y. Tameda, M. Takeda, K. Kadota, D. Ikeda, et al., New air fluorescence detectors employed in the Telescope Array experiment, NIM A, 676 (2012) 54-65

[2] Telescope Array Collaboration, T. Abu-Zayyad et al., The surface detector array of the Telescope Array experiment, NIM A, $\mathbf{6 8 9}$ (2012) 87-97

[3] Pierre Auger Collaboration, A. Aab et al., The Pierre Auger Cosmic Ray Observatory, NIM A, 798 (2015) 172-213

[4] Y.Tameda, et.al, Development of the Cosmic Ray Air Fluorescence Fresnel lens Telescope for a next generation UHECR observatory, Proceedings of 2016 International Conference on Ultra-High Energy Cosmic Rays (2016)

[5] T. Fujii, M. Malacari, M. Bertaina, M. Casolino, B. Dawson, et al., Detection of ultra-high energy cosmic ray showers with a single-pixel fluorescence telescope, Astropart. Phys., 74 (2016) 64-72

[6] T.tomida, et.al., Atmospheric monitor for Telescope Array experiment, EPJ Web of Conferences 53 (2013) 10003 